\title{
Leukocytoclastic Vasculitis
}

\section{A peculiar presentation of scrub typhus}

Anila Vasireddy, ${ }_{1}$ Kanthilatha Pai, ${ }^{2}$ Varsha M. Shetty, ${ }^{3}$ Raviraja V. Acharya, ${ }^{1}$ Ramamoorthi Kusugodlu, ${ }^{1}$ Akhila Doddamani, ${ }^{*}$ Sharath P. Madhyastha ${ }^{1}$

ABSTRACT: Scrub typhus is a disease endemic to the Indian subcontinent caused by the obligate intracellular pleomorphic organism, Orientia tsutsugamushi. Scrub typhus, among other acute febrile illnesses, manifests as prodromal symptoms of fever, malaise, myalgia and anorexia followed by a distinct maculopapular rash, hepatosplenomegaly and lymphadenopathy. We report the case of a patient presenting to tertiary care hospital in southern India in 2021 who developed a rare cutaneous vasculitis secondary to infection with Orientia tsutsugamushi. After performing the Weil-Felix test, a diagnostic titre of >1:640 against OXK was obtained. Furthermore, a skin biopsy was carried out which confirmed the diagnosis of leukocytoclastic vasculitis. The patient was treated with doxycycline and showed drastic improvement in his symptoms.

Keywords: Scrub Typhus, Rickettsia; Vasculitis; Doxycycline; India.

$\mathrm{S}$

CRUB TYPHUS IS A DISEASE ENDEMIC TO THE Indian subcontinent caused by the obligate intracellular pleomorphic organism, Orientia tsutsugamushi. This organism is transmitted naturally through the Leptotrombidium mite population and is accidentally acquired in humans via the bite of a mite as a dead-end host. Scrub typhus, among other acute febrile illnesses, manifests as prodromal symptoms of fever, malaise, myalgia and anorexia followed by a distinct maculopapular rash, hepatosplenomegaly and lymphadenopathy. Rarely, it can also progress to septic shock/multi-organ failure. As in every disease, scrub typhus can have some peculiar presentations. There have been previous case studies reporting features of haemophagocytic syndrome, epididymo-orchitis, acute severe monoarthritis and Guillain-Barré syndrome. ${ }^{1-4}$ In this case report, we present a patient who developed a rare cutaneous vasculitis secondary to an $O$. tsutsugamushi infection with good response to doxycycline.

\section{Case Report}

A 28-year-old male farmer presented to a local primary centre with high grade, intermittent fever $\left(102{ }^{\circ} \mathrm{F}\right)$, vomiting and generalised muscle pain in southern India in 2021 and was treated with antipyretics. Four days later, the patient developed multiple, palpable purpuric eruptions predominantly affecting the lower limbs. Thereafter, the patient was subsequently transferred to a tertiary care hospital in southern India in 2021.

On examination, the patient's vital signs were stable and he was afebrile during the time of presentation at this hospital. A skin examination revealed multiple, palpable purpuric eruptions arranged in a retiform pattern with a dusky necrotic centre and peripheral rim of erythema distributed symmetrically over the bilateral lower limbs [Figures $1 \mathrm{~A}-\mathrm{C}]$. The mucosa, palms and soles were spared. The rest of the systemic examination was deemed to be normal.

A panel of laboratory investigations were carried out to determine the aetiology [Table 1]. The routine urinalysis was unremarkable, confirming no renal involvement. Antinuclear antibody and antineutrophil cytoplasmic antibodies, serum C3 and C4 complement levels were within normal range, making vasculitis due to autoimmune inflammatory disorders less likely.

This constellation of findings is unique to acute febrile illnesses. Therefore, a panel of serum studies were performed to determine the organism. Subsequently, leptospirosis, dengue (non-structural protein 1 antigen and anti-dengue immunoglobulin M), malaria and Hepatitis B and C were ruled out.

However, a Weil Felix test (WFT: tube agglutination) was performed and a diagnostic titre of >1:640 against OXK was obtained. In most cases, a skin biopsy is not routinely performed if clinical and serological criteria for scrub typhus are met. However, due to the rare manifestation of this disease, a skin biopsy was done in order to rule out other aetiologies of vasculitis in the patient.

The skin biopsy done from the lesion revealed necrotising vasculitis [Figure 2]. Hence, the patient was treated with $100 \mathrm{mg}$ of doxycycline twice daily. On day three, improvement of vasculitis and other symptoms were noticeable [Figure 1B]. The patient was discharged on day seven and prescribed with 


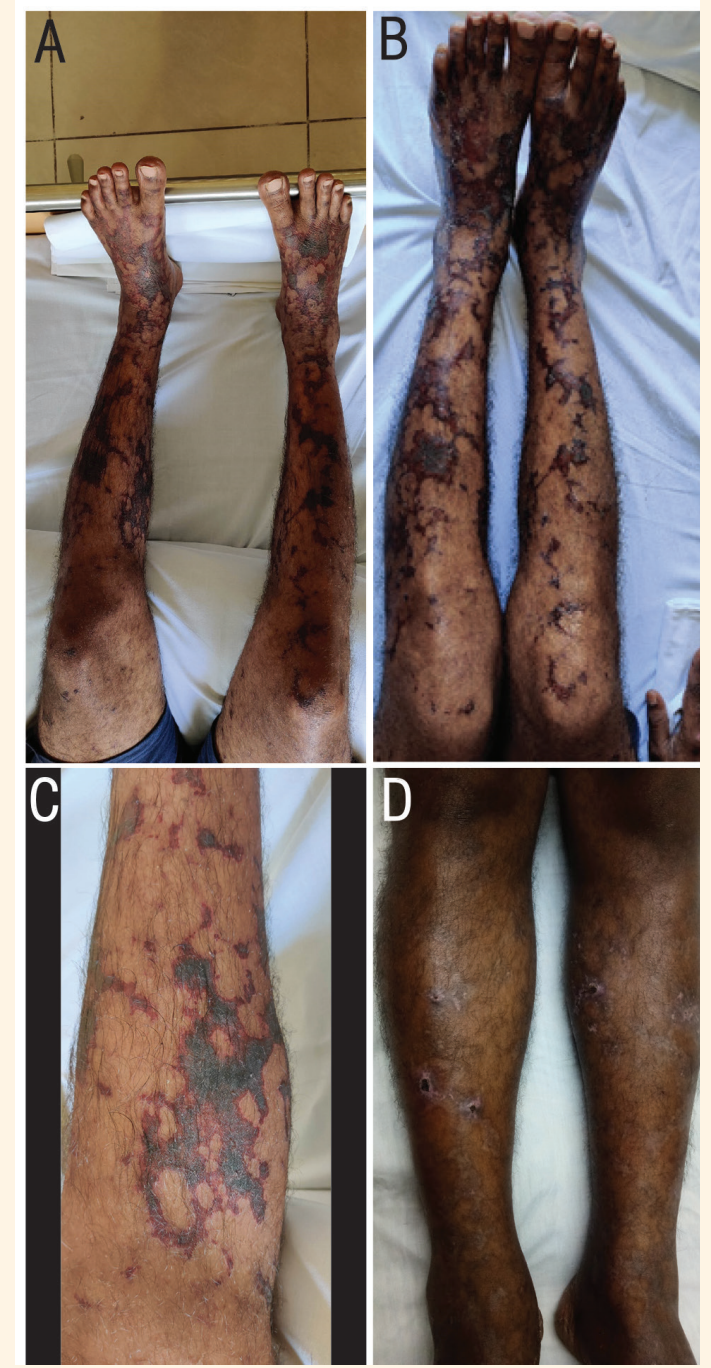

Figure 1: Image showing multiple, palpable purpuric lesions arranged in a retiform pattern with a dusky necrotic center and peripheral rim of erythema distributed symmetrically over bilateral lower limbs on (A) day 1 and (B) day 3. A close-up view of a skin lesion over the left leg on (C) day 1 and (D) a significant improvement in lesions on day 21.

an additional week of doxycycline (100 mg, twice daily). The patient followed up three weeks later with significant improvement in his lesions [Figure 1D].

The patient provided informed consent for the publication of this case.

\section{Discussion}

Scrub typhus is a common cause of pyrexia of unknown origin in India due to its nonspecific clinical features. It is caused by the organism O. tsutsugamushi, which was previously classified under the genus Rickettsia. However, due to different phenotypic and genotypic features, the organism has its own separate genus. In fact, it is a component of the 'tsutsugamushi triangle' formed by Northern Japan/East Russia (North),
Table 1: Laboratory investigation results of a 28 -yearold male patient with leukocytoclastic vasculitis

$\begin{array}{ll}\text { Lab investigation } & \text { Value (normal range) } \\ \text { Haemoglobin in g/dL } & 13.1(13-17) \\ \text { Platelet count in cells/ } \mu \mathrm{L} & 200,000(150,000-400,000) \\ \begin{array}{l}\text { White blood cell count in } \\ \text { cells/ } \mu \mathrm{L}\end{array} & 15,300(4,000-11,000) \\ \begin{array}{l}\text { Aspartate transaminase in } \\ \text { IU/L }\end{array} & 69(5-40) \\ \begin{array}{l}\text { Alanine transaminase in } \\ \text { IU/L }\end{array} & 274(5-40) \\ \begin{array}{l}\text { Alkaline phosphatase in U/L } \\ \text { Erythrocyte sedimentation } \\ \text { rate in mm/hr }\end{array} & 28(40-22) \\ \begin{array}{l}\text { C-reactive protein in mg/L } \\ \text { (430) }\end{array} & 40.92(0-5)\end{array}$

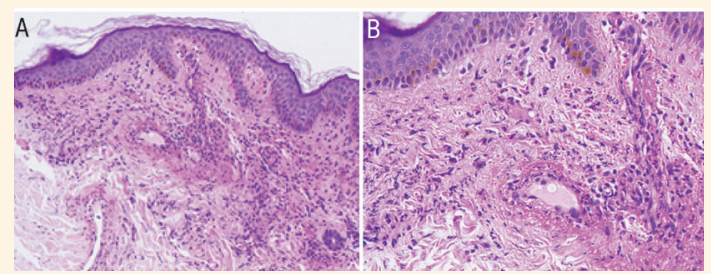

Figure 2: Haematoxylin and eosin stains at $(\mathbf{A}) \times 100$ magnification showing dermis with prominent vasculo-centric infiltrate and $($ B $) \times 200$ magnification of vessels showing fibrinoid necrosis of vessel walls and dense infiltration of vessel walls by neutrophils with leukocytoclasis.

Afghanistan/Pakistan (West) and Northern Australia (South). ${ }^{5}$ There are three strains of O. tsutsugamushi: Karp, Gilliam and Kato. It is important to note that infection with one particular strain does not confer immunity to infection with another strain. ${ }^{5}$

The organism enters the human body via the bite of a chigger mite (trombuculid mite) which multiplies and disseminates through the blood and lymph. Necrosis of the skin occurs at the site of inoculation, forming a black eschar, which is typical of scrub typhus. However, in the Indian subcontinent, a necrotic eschar can only be detected $10 \%$ of the time due to darker skin complexions of the majority of the population and bites located in hidden areas. ${ }^{6}$ Hence, serology or a high degree of clinical suspicion based on epidemiological data must be used to guide the diagnosis. ${ }^{5}$

Scrub typhus usually targets specific populations, typically including those in rural areas predominantly involved in agriculture and those who engage in poor protective personal habits. Moreover, overcrowding plays a huge role in dissemination of the disease as rodents often act as amplifiers of this infection. 
O. tsutsugamushi breaches the endothelial cell barrier creating vascular and perivascular lesions that ultimately cause vascular leakage and end-organ damage to multiple organs in the body. ${ }^{7}$ Once the organism successfully evades the innate defences of the human host, various cytokines such as tumour necrosis factor alpha, interferon gamma (IFN- $\gamma$ ) and macrophage colony-stimulating factor are produced, resulting in the multitude of symptoms experienced by the patient. Both humoral and cellular immunity play a role in combating this organism. It involves the production of antibodies against $O$. tsutsugamushi that can be detected by the WFT and activation of macrophages and helper $\mathrm{T}$ cells (Th1) which secrete IFN- $\gamma^{7}$

The pathophysiology of O. tsutsugamushi causing widespread endothelial damage involves disruption of the adherens junction of the endothelial cells. This results in increased vascular permeability, formation of inter-endothelial gaps, development of actin stress fibres and change in the shape of the endothelial cells from a polygonal to a spindle form. ${ }^{8}$ Furthermore, there is also increased vascular expression of nitric oxide and COX-2 expression by the endothelial cells, resulting in the production of prostaglandins. ${ }^{9}$ In addition, O. tsutsugamushi multiplies within the endothelial cells and, via oxidative stress, destroys the integrity of the blood vessels. ${ }^{10}$ This can be established through immunohistochemical staining of endothelial cells which demonstrates rich deposits of the $O$. tsutsugamushi antigens. ${ }^{11}$

Scrub typhus can occur with a few dermatological manifestations that can overlap with other acute febrile diseases. The typical erythematous maculopapular rash and necrotic eschar can point to a diagnosis of scrub typhus, especially in an area of high endemicity. Moreover, the site of the rash can help differentiate between rickettsial diseases. A maculopapular rash distributed across the trunk, sparing the face, palms and soles makes rickettsial spotted fevers an unlikely diagnosis. ${ }^{12}$ Other tropical diseases with a similar dermatological presentation include dengue, leptospirosis, enteric fever, malaria and melioidosis. ${ }^{13}$

The infectious causes of leukocytoclastic vasculitis are most commonly viral in origin such as hepatitis B (polyarteritis nodosa), hepatitis C (mixed cryoglobulinemia), cytomegalovirus or parvovirus B19. ${ }^{10}$ Parasites, bacteria and rickettsia are lesserknown culprits of systemic vasculitis. It is paramount to determine the underlying cause of vasculitis as this governs the treatment regimen. ${ }^{10}$

The organism is mainly confined to the reticuloendothelial system resulting in hepatosplenomegaly and generalised lymphadenopathy along with other features like fever, myalgia and a centripetally-distributed maculopapular rash. Complications include acute respiratory distress syndrome, acute renal failure, disseminated intravascular coagulation, meningoencephalitis, myocarditis, pericarditis, and acute hearing loss. It is imperative that serology be done in order to ascertain proper treatment. ${ }^{13}$

WFT is a heterophile agglutination test that can be used to diagnose a scrub typhus infection. This test relies on the principle that an antibody triggered by a particular antigen can cross-react with antigens of other species. In this test, antibodies produced by $O$. tsutsugamushi cross-react with the antigen OXK of Proteus mirabilis. For the current patient, a titre of $>1: 640$, which is above the diagnostic titre of 1:320, was obtained. ${ }^{14}$ WFT only tests positive during the second week of the illness and has a low sensitivity and specificity as compared to the indirect fluorescent antibody (IFA) and indirect immunoperoxidase (IIP) tests. Even though IFA/IIP are more accurate and precise, WFT is used because of its cost-effective and swift results. ${ }^{15}$ A recent case report was published linking a case of Henoch-Schönlein purpura to scrub typhus which used indirect immunofluorescence to confirm the diagnosis (IgM antibody: 1:1024). This is an accurate and precise tool to corroborate the aetiology. ${ }^{16}$

The current guidelines for the treatment of scrub typhus includes $100 \mathrm{mg}$ of doxycycline twice daily. Alternatives such as chloramphenicol, azithromycin, tetracycline and rifampicin can be used as secondline options. The treatment with doxycycline renders the patient afebrile within 48 hours. Due to the rarity of the necrotic eschar in the Indian population and nonspecific clinical features, a delay in the start of treatment increases the risk of developing complications and causing irreversible damage. As a result, empirical therapy with doxycycline should be started immediately if there is a high degree of clinical suspicion. ${ }^{17}$

\section{Conclusion}

Scrub typhus, which is often misdiagnosed or underdiagnosed, remains a common zoonotic disease in the Indian subcontinent. This may be due to overlapping clinical features of other tropical diseases, lack of highly sensitive and specific equipment for diagnosis in endemic areas, or delay in presentation. Cutaneous vasculitis secondary to O. tsutsugamushi is an unusual manifestation of this disease and should be considered in endemic areas to avoid delayed treatment. 


\section{AUTHORS' CONTRIBUTION}

$\mathrm{AV}, \mathrm{RVA}$ and SMP conceptualised the report. KP provided the pathology report of the skin biopsy. VMS analysed the skin findings and performed the skin biopsy. AV drafted the manuscript. RVA, RK, $\mathrm{AD}$ and SMP reviewed the manuscript and provided intellectual input. All authors approved the final version of the manuscript.

\section{References}

1. Lin $\mathrm{M}$, Huang A, Zheng X, Ge L, He S. Misdiagnosis of scrub typhus complicated by hemophagocytic syndrome. BMC Pediatr 2019; 19:102. https://doi.org/10.1186/s12887-019-1475-x.

2. Saha A, Sarkar S, Patil A, Besra B, Saha C. Epididymo-Orchitis in Scrub Typhus. Indian J Pediatr 2018; 85:1035-6. https://doi. org/10.1007/s12098-018-2682-4.

3. Handattu K, Yellanthoor RB, Konda KC, Kini S. Acute severe monarthritis: a rare manifestation of scrub typhus. BMJ Case Rep 2018; 11:bcr2018227002. https://doi.org/10.1136/bcr-2018227002 .

4. Sawale VM, Upreti S, Singh TS, Singh NB, Singh TB. A rare case of Guillain-Barre syndrome following scrub typhus. Neurol India 2014; 62:82-3. https://doi.org/10.4103/0028-3886.128340.

5. Chogle AR. Diagnosis and treatment of scrub typhus--the Indian scenario. J Assoc Physicians India 2010; 58:11-2.

6. Abhilash KPP, Gunasekaran K. Eschar: A vital clue for diagnosis of scrub typhus. Curr Med Iss 2019; 17:134-7.

7. Chakraborty S, Sarma N. Scrub Typhus: An Emerging Threat. Indian J Dermatol 2017; 62:478-85. https://doi.org/10.4103/ijd. IJD_388_17.
8. Valbuena G, Walker DH. Infection of the endothelium by members of the order Rickettsiales. Thromb Haemost 2009; 102:1071-9. https://doi.org/10.1160/TH09-03-0186.

9. Kumar ASP, Prasad AM, Gumpeny L, Siddappa R. Leukocytoclastic vasculitis and polyarthralgia in scrub typhus: An unusual presentation. Int J Med Public Health 2013; 3:352-4. https://doi. org/10.4103/2230-8598.123530.

10. Lakshmanan S, Seetharaman K, Sr R, Sivanandam S, Ak K. Scrub typhus vasculitis causing pan-digital gangrene. Int J Case Rep Images IJCRI 2015; 6:416-21. https://doi.org/10.5348/ijcri201569-CR-10530.

11. Kim DM, Park CJ, Lim SC, Park KH, Jang WJ, Lee SH. Diagnosis of scrub typhus by immunohistochemical staining of Orientia tsutsugamushi in cutaneous lesions. Am J Clin Pathol 2008; 130:543-51. https://doi.org/10.1309/X17HNNJKMYGHT4HP.

12. Rajagopal R, Khati C, Vasdev V, Trehan A. Scrub typhus: A case report. Indian J Dermatol Venereol Leprol 2003; 69:413-5.

13. Wang CC, Liu SF, Liu JW, Chung YH, Su MC, Lin MC. Acute respiratory distress syndrome in scrub typhus. Am J Trop Med Hyg 2007; 76:1148-52. https://doi.org/10.4269/ajtmh.2007.76.1148.

14. Cox AL, Tadi P. Weil Felix Test. From: https://www.ncbi.nlm.nih. gov/books/NBK559225/ Accessed: Sep 2021.

15. Janardhanan J, Trowbridge P, Varghese GM. Diagnosis of scrub typhus. Expert Rev Anti Infect Ther 2014; 12:1533-40. https://doi. org/10.1586/14787210.2014.974559.

16. Im JH, Choi SJ, Chung MH, Lee SY, Park YK, Kwon HY, et al. A case of Henoch-Schönlein purpura associated with scrub typhus. BMC Infect Dis 2020; 20:286. https://doi.org/10.1186/s12879-02005001-x.

17. Mahajan SK. Scrub typhus. J Assoc Physicians India 2005; 53:954-8. 